\title{
LAPAROSCOPY
}

\section{THE EYE THROUGH THE CLOSED DOOR \\ IS IT SAFE IN GYNECOLOGIC EMERGENCY?}

\author{
Mohamed Salama Gad, M.D. \\ Obstetrics \& Gynecology Department, El-Menoufiya University
}

\section{ABSTRACT}

Introduction : Laparoscopy is one of the commonest operations in modern gynaecology and it has the potential to be used either as a therapeutic or diagnostic modality. The inherent risks during laparoscopy may add to the risks of basic procedures especially in emergencies.

Objectives: To evaluate the role of laparoscopy versus its complications in women presenting with acute pelvic pain (APP) with uncertain diagnosis as gynecologic emergency.

Design : Retrospective observational clinical study.

Setting : Endoscopy unit in Obstetrics and Gynecology Department, El-Menoufiya University.

Methods : We have carcfully reviewed and analyzed the medical records of 869 consecutive cases admitted for laparoscopy during a three-year period. The incidence, indication, nature, causc, and operative sheet details were recorded with special attention 10 complications in 40 women who presented with APP as an emergency.

Results : Gynecologic disorders were diagnosed and treated in $60 \%$ of cases and surgical disorders in $20 \%$. Total complication rate was $30 \%$. There were $30 \%$ operative, $12.5 \%$ anesthetic, $7.5 \%$ nursing and $25 \%$ equipment complications. The complications were graded as mild in $70 \%$ moderate in $25 \%$ and severe in $5 \%$ and there were no deaths.

\section{Conclusions :}

Laparoscopy is an ideal and useful as a diagnostic and therapeutic tool in gynecologic emergencies. However History and careful physical examination together with ultrasound are mandatory to good paticnt care before laparoscopy.

Laparoscopic complications increase with increasing complexity of surgery and in emergency setting it is never too late to change to laparotomy if the condition is demands or if the surgeon's skill is insufficient.

Rigid maintenance of equipment, proper patient selection, well trained operating room personnel and continuing medical education can help to avoid complications, develop and maintian excellence.

Key words : Laparoscopy, Gynecologic Emergency, Complications, and Acute Pelvic Pain.

\section{INTRODUCTION}

Gynecologists are faced increasingly with the diagnosis and management decisions in any patient seen in emergency situations. Acute pelvic pain (APP) is a common presentation in gynecology. APP definition is arbitrary and the incidence of its different etiologies is difficult to estimate. Often the duration of APP is only a few hours, but it can be days. It usually presents with a sudden onset, but may be insidious (1).

Careful history taking as well as skill in physical diagnosis together with understanding the management considerations of the APP is critical to good patient care. Inspite of that, the etiology remains unclear in some cases ${ }^{(2)}$. Until recently, the age-old surgical policy of "not letting the abdominal wall get between you and the diagnosis" 
has proved more challenging as physicians attempt to diagnose patients with abdominal pain ${ }^{(3)}$. Laparotomy or laparoscopy is the 100\% effective approach for penetrating the "wall". The development and rapidly advancing technology and increased experience with laparoscopy decrease patient morbidity and hospital costs ${ }^{(4)}$. However, every surgical procedure including laparoscopy has complications and most can be avoided by applying the basic principles of good surgical techniques.

Laparoscopy was proposed as an ideal surgical emergency procedure as it is a minimally invasive surgery, safe and quick diagnostic devise. Moreover, in gynecology, laparoscopy has been widely used for diagnosis and treatment of acute conditions including APP(1).

Laparoscopic surgery is associated with a small but significant double risk of complications. The first risks inherent in the specific operation, and the second are risks of the laparoscopic mode of access which would not have occurred if traditional surgery had been selected. It may be reasoned that as the complexity of minimal invasive surgical procedure increases, so will be the number of complications $^{(5)}$.

The authors of the American Association of Gynaecologic Laparoscopists (AAGL) membership stated, no data are available to compare the therapeutic efficiency of laparoscopy and laparotomy. Meanwhile, the true incidence of laparoscopic complications is unknown since the vast majority are not reported (6). On the same time many publications about complications are from specialized centers in laparoscopic surgery and may not be representative of the situation in smaller centers.
A multicenter study from a number of leading laparoscopic French centers, indicated that the risk of complications was related to the experience of the operator. A prior laparotomy also increased significantly the risks of subsequent laparoscopy. The extent of these complications varies greatly from the minor to the major and may even prove fatal.

Complications may be associated with every step of the laparoscopic procedure. The complication rate is increased as would be expected, when surgical operativeprocedures are performed. The necessary dissection, ligation, division, and use of energy sources such as electricity or lasers, all pose risks ${ }^{(7)}$.

Severe laparoscopic complications are adverse outcomes and can lead to considerable physical and emotional suffering for patients, relatives, and doctors as well as vast financial costs and almost inevitably to litigation.

As laparoscopy can be used to solve the problem of diagnosing difficult cases of abdominal diagnostic dilemmas, this study was designed to evaluate the role of laparoscopy versus its complications in women presenting with APP with uncertian diagnosis as a gynecologic emergency.

\section{MATERIALS \& METHODS}

Setting: Gynaecologic Endoscopic Unit in Obstetrics and Gynecology Department, El-Menofiya University Hospital.

Plan: The medical records of 869 women in the fertile age period who were admitted for laparoscopy during a three-year period (Between May 1999 and April 2002), were revised for the indications. There were 40 consecutive women who complaind of acute pelvic pain (APP) 
underwent operative laparoscopy because their diagnoses were uncertain. Their medical records were reviewed and analyzed retrospectively. Informed written consent for both endoscopic and open surgery was routinely obtained and included in each file.

For all selected cases, the following protocol was applied:

History: Systematic history was mandatory including patient complaint, full pain description, radiation and its relation to menstrual cycle. Also, date of last menses, regularity of the periods, contraception, any vaginal discharge, and previous operations were asked about. Furthermore, gastrointestinal and urological symptoms were also inquired about.

Examination: A careful physical examination was done including precise description of pain location, site of a scar tissue, and careful gentle gynecologic examination within the limits of the patient pain tolerance. General surgery consultation was routinely done for all cases.

\section{Investigations: 2 types were done:}

A-Primary routine ones including mid-stream urine specimen, swabs (high vaginal and endocervical) for microorganisms, complete blood count, and urinary or serum pregnancy test $(\beta \mathrm{HCG})$ were done as indicated

B- Ultrasound scan : vaginal / abdominal, was mandatory for all cases.

Depending on the results obtained, laparoscopy was done within a short period of time.

\section{Technique of Surgical Laparoscopy:}

Equipment for emergency laparotomy was ready in the theatre before the start. Laparoscopy procedures were done under general endotracheal anesthesia with urinary catheter and nasogastric tube routinely inserted. After low flow $\mathrm{CO}_{2}$ insufflation until 12-14 $\mathrm{mmHg}$ pressure, 10-mm trocar was inserted just below the umbilicus, through which the telescope was placed and used as a camera port. A complete initial systematic survey of the abdomen was done; and to improve visceral pelvic examination, Trendelenberg position was used. Accessory $4 \mathrm{~mm}$ trocars for organ manipulation were then inserted as needed under direct sight control. Excellent visualization of the pelvic organs especially tubes, uterus and appendix was achieved

From each patient's record file, the following data were obtained:

- Biodata of the women (age, parity, height, weight, body mass index and previous laparotomies).

- The indication of laparoscopy and the operation time.

- The operative sheet details with special attention to the complications \& the surgeon's experience. All laparoscopic complication were classified according to the system proposed by Chapron et al, in $2002^{(7)}$.

Mild complications: transient and need no specific therapy.

Moderate complications: encountered and treated during laparoscopy.

Severe complications: unintended laparotomy (open surgery conversion) during laparoscopy.

Comparison of percentages was done using the chi-square test. Comparison of means was done using analysis of variance. The thereshold of significance was fixed at $5 \%$ level.

We are using guidelines for safe endoscopic surgery in our Gynaecology Endoscopic Unit for minimizing complications during operative laparoscopy. The guidelines used are those summarized by Smith $1992^{(8)}$. 
Guidelines for minimizing complications during operative laparoscopy. (8)

- During anaesthesia:

- Use cuffed endotracheal tube

- Use nasogastric drainage.

- Avoid overforceful mask ventillation

- Use complete muscle paralysis

- In positioning patient:

- Place in frog-leg lithotomy position.

- Avoid excessive pressure on inner thighs.

- Avoid excessive hip or knee flexion or extension.

- Use soft shoulder padding, if any.

- Use knee-and foot-supporting stirrups.

- Empty and continuously drain bladder

- Maintain arm on surgeon's side (usually the left) parallel alongside the body.

- Avoid excessive Trendelenburg's position.

- Lower operating table to level of the surgeon's elbows or hips to maximize control during insertion of umbilical or auxilliary trocars.

- In establishing pneumoperitoneum:

- Percuss left-upper quadrant to detect gastric distension.

- Elevate umbilical skin before making skin incision.

- During Veress needle insertion:

* Test spring mechanism before placement.

* Leave valve open.

* Direct to hollow of the sacrum.

* Advance only $2-3 \mathrm{~mm}$ after piercing the parietal peritoneum.

* Perform saline aspiration test.

- Do not insufflate $\mathrm{CO}_{2}$ at more than 1 liter/min initially.

- If no loss of dullness to percussion over the liver edge is observed after insufflation of 1 liter of
$\mathrm{CO}_{2}$ suspect peritoneal or omental insufflation.

- Avoid overinsufflation of the abdominal cavity; generally maintain intraabdominal pressure at less than $16 \mathrm{mmHg}$.

- During laparoscope insertion and withdrawal:

- During trocar insertion :

* Maintain patient horizontal.

* Extend index finger to within $3 \mathrm{~cm}$ of trocar tip to protect against sudden deep penetration.

* Use controlled twisting motion.

* Direct trocar tip toward sacral hollow.

* Advance no more than $2 \mathrm{~cm}$ beyond parietal peritoneum.

- When withdrawing trocar sheath, replace laparoscope (not trocar) first, after emptying the abdominal cavity of excess $\mathrm{CO}_{2}$.

- During auxilliary trocar insertion:

- Transilluminate for visualization of epigastric vessls.

- Identify at laparoscopy the inferior epigastrics on the anterior lateral to the umbilical artery remnants; they usually arise just medial to where the round ligaments enter the internal iguinal ring.

- Place trocars as high above the symphysis as cosmetically possible, but never less than $3 \mathrm{Cm}$.

- Insert under dirct laparoscopic visualization.

- Direct downward, toward uterine fundus, not laterally.

- If peritoneum tents around trocar tip, direct cranially along the anterior abdominal wall into the umbilical sleeve.

- Consider radially expanding sleeves when large-diameter trocars and sleeves must be placed, especially laterally. 


\section{- During endoscpic surgery:}

- Minimize use of unipolar electrocautery.

- Disconnect or turn off all electrosurgical or laser units when not in use, even temporarily.

- Identify ureters before any surgery on the pelvic side wall.

- Mobilize the ovaries completely before performing a cystectomy.

- Minimize forceful blunt dissection, especially when adhesions involve bowel serosa.

- Use traction/ countertraction to identify tissue planes.

- Spread jaws of scissors to develop tissue planes, in lieu of cutting across the tissues.

- Avoid cautery of bowel serosa.

- Cauterize/ coagulate vessels before transection.

- Avoid scissor action ("crossed swords") between different instruments to minimize the risk of pinching or traumatizing bowel or omentum.

- Do not cut any tissue before fully identifying the anatomy.

\section{RESULTS}

A total of 869 laparoscopies were performed during the study period. APP with uncertain diagnosis in the study group represented $4.6 \%$. The biodata of the 40 women included in the study were shown in table (I). All were during the fertile age. Obesity was encountered in 10 cases (25\%); it was diagnosed if BMI $>26$. Previous surgery was reported in 12 women (30\%). These included appendectomy in 5 cases, ovarian cystectomy in 3 cases, previous CS in 2 cases, ectopic pregnancy in 1 case, and myomectomy in 1 case.

Table (II) shows the laparoscopic findings in the study group as underlying etiologies for APP.
Gynecologic disorders were found in $24 / 40$ cases $(60 \%)$, surgical disorders in $8 / 40$ cases $(20 \%)$, pelvic adhesions in $4 / 40$ cases (10\%) and no pathology was encountered in 4 cases.

Table (III) shows the emergency procedures done using laparoscopy. Correct diagnosis could be established in 36/40 cases (90\%). Laparoscopic surgeries (minimally invasive procedures) had been performed in 33 out of 40 cases ( $82.5 \%$ ). Open surgery conversion occurred in $5 / 40$ cases (12.5\%), 2 cases to deal with major complication (5\%) and in 3 cases for appendectomy by surgeons.

Table (IV) shows the nature and grades of complications encountered during the study. They were grouped as anaesthetic in (5/40) of the cases $(12.5 \%)$, nursing in $(3 / 40)$ of cases $(7.5 \%)$, operative in (12/40) of the cases (30\%), and equipment in (10/40) of cases $(25 \%)$.

Figure (1) shows the different grades of complications. They were mild in (28/40) (70\%) of cases, moderate in (12/40) (25\%) of cases, and severe in $(2 / 40)(5 \%)$ of cases. Mild complications required no specific therapy. Moderate compliction were encountered during laparoscopy and dealt with safely. Severe complications that needed laparotomy to deal with direct bowel trocar trauma in one case and to control tubal mesentry haemorrhage in another case. The 2 cases had abdominal scar and pelvic adhesions. There were no deaths, postoperative ileus, fever, thrombophlebitis, transfusions, urinary tract infection, ureteral injuries or atelectasis. None of the patients was admitted or readmitted because of complications. The 3 cases of open surgery conversion for appendectomy were not recorded as complications. 
Table I : The biodata of patients included in the study group. $(n=40)$

\begin{tabular}{|l|c|c|}
\hline & Range & Mean \pm S. D. \\
\hline Age (years) & $20-39$ & $29.5 \pm 3.86$ \\
Parity & $0-8$ & $2.0 \pm 1.1$ \\
Height (cm) & $145-164$ & $154.1 \pm 4.7$ \\
Weight (kgm) & $45-84$ & $65.6 \pm 7.2$ \\
Body Mass Index* & $22.6-34.9$ & $26.9 \pm 3.9$ \\
\hline
\end{tabular}

* BMI was $>26$ in 10 cases in the study.

Table II : Findings at laparoscopy

\begin{tabular}{|l|c|c|}
\hline \multicolumn{1}{|c|}{ Pathology } & Number of cases (Total 40) & $\%$ \\
\hline Gynecologic disorders: & $(\mathrm{N}=24)$ & 60 \\
- Ruptured ovarian cyst & 5 & \\
- Twisted ovarian cyst & 2 & \\
- Pelvic inflammatory disease & 6 & \\
- Endometriosis. & 4 & \\
- Intact ectopic pregnancy & 4 & 20 \\
- Uterine perforation & 3 & 10 \\
Surgical disorders: & $(\mathrm{N}=8)$ & 10 \\
- Acute appendicitis. & $(\mathrm{N}=4)$ & \\
Pelvic adhesions & $(\mathrm{N}=4)$ & \\
No pathology detected: & & \\
\hline
\end{tabular}

Table III : Emergency procedures done using laparoscopy

\begin{tabular}{|l|c|c|c|}
\hline \multicolumn{1}{|c|}{ Pathology $(\mathbf{n}=\mathbf{3 2})$} & DL & Mis & OSC \\
\hline Ruptured ovarian cyst & 5 & 5 & 0 \\
Twisted ovarian cyst & 2 & 2 & 0 \\
Pelvic inflammatory disease & 6 & 6 & 0 \\
Endometriosis & 4 & 4 & 0 \\
Intact ectopic pregnancy & 4 & 4 & 0 \\
Uterine perforation & 3 & 3 & 0 \\
Acute appendicitis & 8 & 5 & 3 \\
Pelvic adhesions & 4 & 4 & 2 \\
\hline Total : No. $(\%)$ & $36(90 \%)$ & $33(825 \%)$ & $5(12.5 \%)$ \\
\hline
\end{tabular}

$\mathrm{DL}=$ Diagnostic Laparoscopy

MIS = Minimal Invasive Surgery

$\mathrm{OSC}=$ Open Surgery Conversion 
Table IV : Nature and grades of laparoscopic complications.

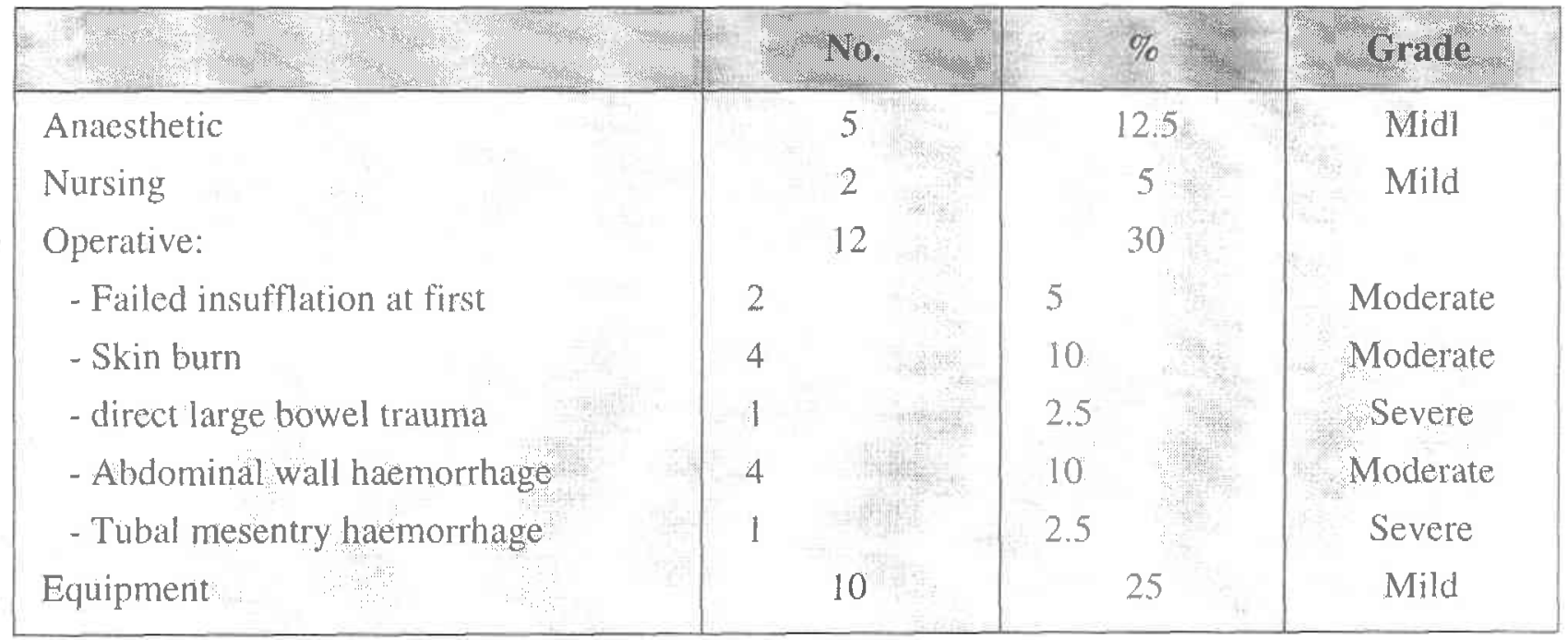

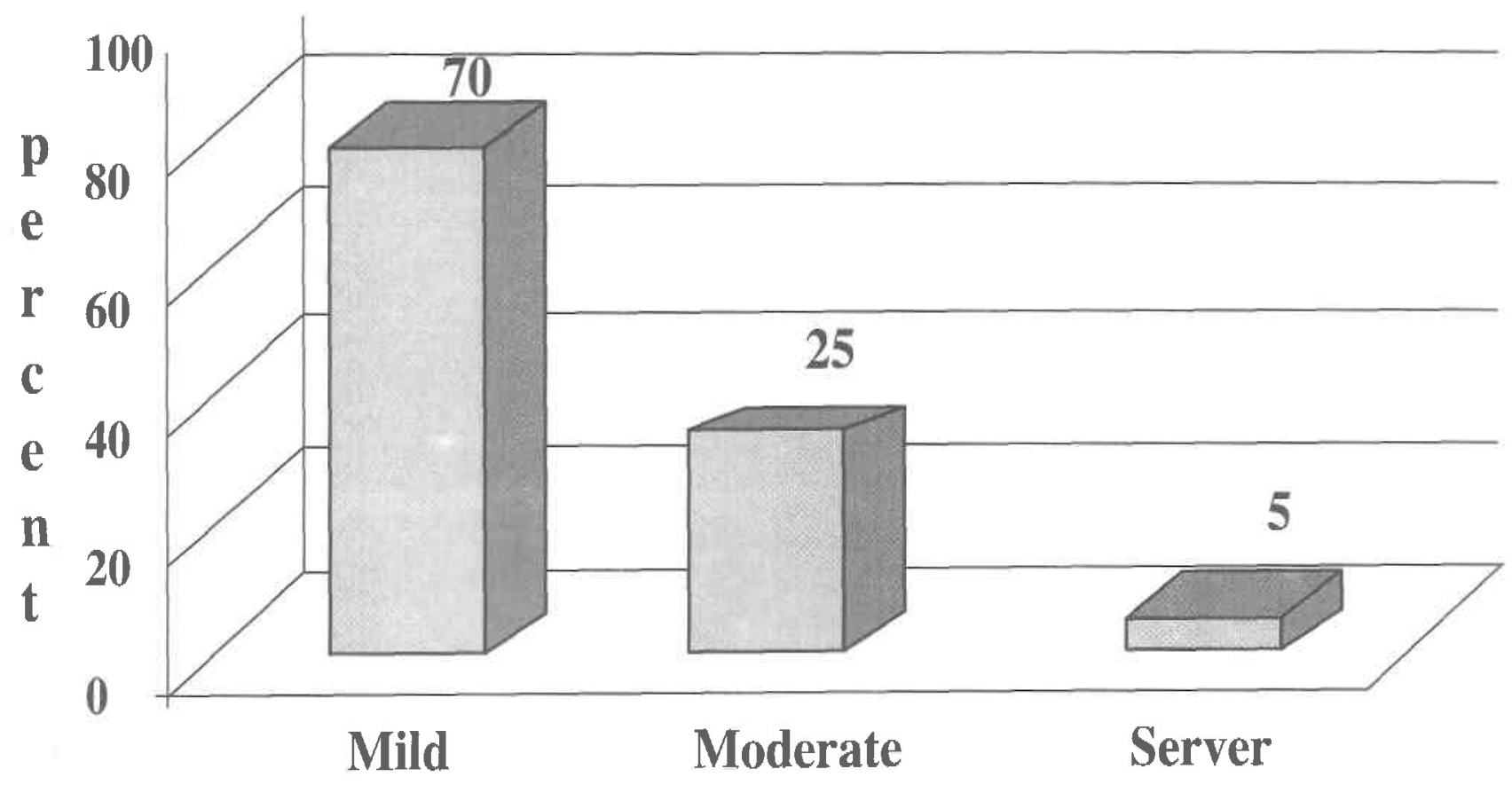

Fig. 1. Grades of laparoscopic complications 


\section{DISCUSSION}

APP is one of the most frequent causes of gynecologic emergencies in developed countries (9). In the present study, APP incidence was $4.6 \%$ and laparoscopy was performed because the clinical finding and results of investigations including ultrasound were insufficient to establish the diagnoses even after a period of observation. After laparoscopy the diagnosis could be reached in $90 \%$ and gynecologic disorders were diagnosed in $60 \%$ of cases. Usually in gynecologic cases with APP, clinical assessment, laboratory findings and ultrasound, provide preoperative diagnosis. Borderline situations can delay an early accurate treatment, so laparoscopy is useful to define doubtful diagnostic questions ${ }^{(2)}$.

Laparoscopy has a high accuracy degree and has been found to be a sensitive procedure in APP diagnosis limiting the number of "unnecessary laparotomy" that remains between $5-14 \%$. On the other hand, skilled laparoscopic teams can treat gynecologic emergencies with a minimally invasive approach ${ }^{(6)}$. Inspite of that, when doing an emergency laparoscopy; open laparotomy instrumentation should be immediately available and standby in the operating room; a delay for laparotomy should never happen. In our series as shown in table (II), minimal invasive surgery was done in $82.5 \%$ and open surgery conversion for appendectomy was done in $7.5 \%$. In an emergency setting, conversion must not be considered a complication, merely a change of the incision $\operatorname{size}^{(1)}$.

In this work, pelvic inflammatory disease was diagnosed in 6 women (15\%) and culture materials were obtained during laparoscopy without open surgery. All cases were treated and responded well to medical treatment. It is found that clinical diagnosis of PID was no better than chance, laparoscopy alone had a sensitivity of $50 \%$ with specificity of $80 \%(10)$. In United States, laparoscopy is reserved for women in whom the clinical diagnosis of PID is uncertain, or when therapy is unresponsive in 48 hours $(3$ )

Sensitive $\beta$-Subunit pregnancy test, and vaginal ultrasound detection of ectopic pregnancies, even prior to the onset of APP, have made the diagnosis and management of the unruptured ectopic pregnancy a semi-elective laparoscopic procedure (11). In this study, unruptured ectopic pregnancy was found in $10 \%$ and treated successfully by laparoscopy. However the gold surgical standard is to perform an immediate laparotomy to stop bleeding and save surgical time rather than to do laparoscopy once pelvic pain suggests a rupture of an ectopic pregnancy in the presence of unstable vital signs with documented haemoperitoneum ${ }^{(12)}$

Acute appendicitis is the most common surgical emergency of the abdomen in the United States (13). With diagnostic laparoscopy, a "negative" appendicitis can be very useful to the female patient, since through abdominal and pelvic exploration at laparoscopy can positively establish the reason for APP. Because of the relation of the appendix and the pelvic organs, a gynecologist was the first to describe laparoscopic appendectomy (14). In this study, acute appendicitis was found in $20 \%$ of cases. Five $(12.5 \%)$ of whom underwent laparoscopic appendectomy by a surgeon and open surgery conversion occurred in three cases $(7.5 \%)$. Pathologic examination documented acute appendicitis in all cases. Open surgery conversion to do appendectomy was not recorded as a complication for two reasons; first it is not directly related to laparoscopy and second appendicitis is a surgical diagnosis. 
Ruptured small benign ovarian cyst was found in $7.5 \%$ and required no further emergency treatment. Twisted ovarian cyst was found in $5 \%$ where laparoscopic ovarian cystectomy was done safely and histopathology examination revealed simple cyst. Endometriosis was diagnosed in 10\% and laparoscopic electrocautery evaporization was done for all visible spots.

The most interesting three cases $(7.5 \%)$ in the study were APP due to uterine perforations, two of them were post evacuation and APP occurred on the same day. Ultrasound revealed incomplete evacuation together with free fluid in Douglas pouch (DP). Laparoscopy diagnosed uterine perforation with free blood in DP. Suction-irrigation was done together with spray coagulation at the perforation sites. Curettage was done under laparoscopic control. The other third perforation was due to IUCD insertion of $\mathrm{Cu}-\mathrm{T}$ 380 , that was seen partially projecting from the uterine wall. The IUCD was removed safely via hysteroscopy under laparoscopic control. APP suddenly occurred post insertion and the IUCD was removed on the same day.

Laparoscopic surgery causes a small but significant risk of complications ${ }^{(6)}$. The occurence of complications during laparoscopy depends upon the experience of the operator and the level of complexity of the surgery. Complications are an inevitable part of surgeon's career. so surgical skills should be aquired gradually to develop the necessary hand-eye co-ordination during low risk situations. Every competent gynaecologist must be familiar with laparoscopic complications and can deal with them. Refining personal opearative technique is critically important ${ }^{(15)}$, and knowing your limits is equally important for minimizing complications. Many complications are caused by momentary attention lapses; some are inevitable, an some are the result of poor judgement ${ }^{(8)}$.

In this study, the total complication rate was $30 \%$. These results are relatively high when compared to others. The reasons may be explained by the fact that all cases included were emergencies, their numbers were small, our endoscopic unit is still growing and the system used for complication grading may be different. It is reported that a total laparoscopic complication rate of $1.54 \%$ in 13336 routine procedures ${ }^{(16)}$ may be acceptable. In 2002, a complication rate of $2.76 / 1000$ was reported. The investigator defined complications as unintended laparotomies ${ }^{(7)}$. In our study, all complications were diagnosed during or at the end of laparoscopy and were treated immediately. the anesthetic complications were transient and resulted from fluid overload, and this problem was corrected by limiting the intraoperative fluid to a maximum of $1500 \mathrm{~mL}$ regardless of urine output or duration of the procedure (17). Improper anaesthetic techniques may contribute to the development of endoscopic complications. Complete muscle relaxation must be maintained at all times during operative laparoscopy procedures. Nasogastric tube should be considered for any patient in whom there is a question of gastric enlargement by air or liquid ${ }^{(8)}$. The AAGL survery in 1991 noted that complications of anesthesia are rare during laparoscopy (1.8/100000), and more than $50 \%$ of anesthesia related deaths are related to hypoventillation $^{(18)}$.

Obesity and abdominal scars were considered as relative contraindications for closed laparoscopy in the past. In our study, obesity was diagnosed by BMI $>26$ in 25\% and previous abdominal scars were present in $30 \%$ of cases. The safe closed $\mathrm{Z}$ 
entry under vision of Semm recommendations reported easy success in all cases except two. Although failure to complete the laparoscopic procedure rarely causes significant morbidity. It shoud be regarded as a complication during emergency. By far the most common reason of failure is the inability to establish a proper pneumoperitoneum ${ }^{(16)}$. This tends to occur in the very obese patients or when the tentative or inexperienced surgeon fails to place the Veress needle in the proper space. The majority of laparoscopic complications (whether operative or diagnostic) occur during placement of the Verress needle or the trocars ${ }^{(19)}$. A variety of techniques are described to minimize the potential for incorrect needle placement, including listening for the "hissing" sound, the aspiration test, the "hanging up" method, and the reading of the intraabdominal pressure ${ }^{(14)}$. In this study, we had 2 cases of difficult insufflation and the reason was obesity in one case and extensive adhesions in the second case.

It is widely accepted that the most dangerous moment in laparoscopic surgery is during the insertion of the sharp primary trocar that is almost invariably performed in a blind fashion (20). Injuries to the large bowel caused by a trocar are serious and require laparotomy. Small, 1-2 cm lacerations, may be treated by primary closure $(21)$. In this study, there was one case of trocar perforation, "sub-umblical $10 \mathrm{~mm}$ one", to transverse colon. The patient was a case of secondary infertility with a history of previous laparotomy by a longitudinal scar for myomectomy. Pneumoperitoneum occurred without complication. On inserting of the telescope, intestinal mucosa was seen. the trocar was kept in situ and laparotomy was done for primary repair. So if it is anticipated that large bowel is at risk, the bowel should be prepared preoperatively with GoLYTEL. Adhesions from the bowel should never be torn free but rather divided. Electrical energy should not be used in close proximity to the bowel ${ }^{(22)}$.

Correct maintenance of telescopes, cameras and light cables will ensure optimul visualization during all endoscopic procedures. Good irrigation and suction devices are essential to identify normal anatomy and sites of bleeding. Backup equipment is not a luxury but essential in the event of accidental damage (12). During this study equipment failure was present in $25 \%$ and involved dysfunction of the bipolar cautery because of breakage, electric cord failure, or fouling of tips. Other equipment failure included video dysfunction. Attendance of a biomedical engineer in the theatre will help to resolve most of the equipment complications. Continuous endoscopic equipment refinement is essential. Equipment checks will prevent the frustration of failure and encourage safe practice.

Nursing complications were graded as mild and occurred in 5\% and resulted from improper use of the Allen stirrups, leading to transient femoral and peroneal neuropathy. Nerve stretch is the most common type of injury associated with laparoscopy. Motor and sensory deficits usually appear immediately after surgery, progress for several weeks and resolve over the following 3 to 9 months (23).

Vascular injuries accounted for $30 \%$ to $50 \%$ of surgical trauma at laparoscopy. In a survey of 100000 laparoscopic procedures reported only 34 injuries to the major internal blood vessels $(0.34$ per 1000 cases) ${ }^{(5)}$. According to the AAGL data, the vascular major complications are much more common today. Verress needle accounts for about 
$36 \%$ of vascular injuries and the primary and auxillary trocars account for about $32 \%$ each ${ }^{(24)}$.

Additional bleeding can occur during the course of operative laparoscopic procedures. So, high-risk situations should be identified and meticulous adherence to the details of proper technique is essential to prevent complications. In this study, abdominal wall haemorrhage occurred in $10 \%$ and were managed by occlusive pressure and overnight hospitalization. In one case, tubal mesentery hemorrhage occurred and the bleeding could not be controlled, so laparotomy was done. Abdominal scar and extensive pelvic adhesions were risk factors in that case.

Before ending the discussion, it is valuable to say that the endoscopic surgery does present new challenges. An endoscopic approach aims to provide benefits to the patient, surgeon and health service providers. What role laparoscopic surgery can play in the treatment of APP has yet to be decided. Whether this approach takes its place just as an alternative with the other standard procedures or becomes the procedure of choice, will be answered only after several years of follow up. Further studies and documentation are needed of such factors as costs saved, safety and efficacy which will serve to establish which is better for emergency, laparoscopy or laparotomy (25).

In most countries endoscopic surgery has been introduced by innovative surgeons without clinical validation and with outcome data limited to uncontrolled personal series or retrospective comparisons with equivalent results to open surgery. In the era of evidence based medicine the triad of controlled clinical trials, reporting of complications to an official central body and proof of safety must be fullfiled.

\section{CONCLUSIONS}

- Laparoscopy is an ideal and useful approach as a diagnostic and therapeutic tool in gynecologic emergencies. However systematic history and careful physical examination together with ultrasound are mandatory to good patient care before laparoscopy.

- Laparoscopic complications increase with increasing complexity of surgery and in emergency setting it is never too late to change to laparotomy if the condition needs it or if the surgeon's skill is insufficient.

- Rigid maintenance of equipment, proper patient selection, well trained operating room personnel and continuing medical education can help to avoid complications, and develop and maintain excellence.

\section{REFERENCES}

I- Knudsen, U. B.; and Agaard, J. "Acute pelvic pain". In: Studd, J. (ed.), Progress in obstetrics and Gynecology, 13 Churchill Livingstone; (1998) 21: 311-321.

2- Maione, G.; Forti, D.; Fontona, A. et al.: "The emergency laparoscopy". Min Invas Ther and Allied Technol; (1996) 5: 51-57.

3- Silen, W : "Cope's early diagnosis of the acute abdomen". 19th Ed. Rev. New York, Oxford University Press (1996).

4- Phillip, P.; Mullins, D.; and Erwin, t.: "Role of laparoscopy in the evaluation of abdominal trauma." Am. J. Surg; (1993) 166: 707-709.

5- Corfman, R. S.; Diamond, M. P.; ad DeCherney, A. (eds): Complications of Laparoscopy and Hysteroscopy. Cambridge : Blackwell Scientific (1993). 
6- Hasson HM, Rotman C, Rana $\mathrm{N}$, et al : Open laparoscopy; 29 year experience. Obstet Gynecol; (2000) 96: 763-766.

7- Chapron C, Faurconnier A, goffinet F, et al,: Laparoscopic surgery is not inherently dangerous for patients presenting with benign gynecological pathology. Hum Reprod; (2002) 17: 1334-1342.

8- Smith, S. : "Minimizing, recognizing, and managing laparoscopic complications". In: Murphy, A, and Azziz, R. (eds), Operative Laparoscopy and Hysteroscopy, Spirnger; (1992) 25: 248-266.

9- Rigg, L. M. : "Emergency medicine: A vigorous new specialty". N. Engl. J. Med.; (1981) 304: 480-3.

10- Sellors, J.; Mahony, J.; Goldsmith, C. et al.: "The accuracy of clinical findings and laparoscopy in pelvic inflammatory disease." Am. J. Obstet. Gynecol.; (1991) 164: 113-120.

11- Sultana, C. J.; Easley, K.; and Collins, R. L.: "Outcome of laparoscopic versus traditional surgery for ectopic pregnancies." Fertil. Steril.; (1992) 57: $285-289$.

12- Gray, D. T.; Thorburn, J.; Lundorff, P. et al.,: "A cost effectiveness study of a randomized trial of laparoscopy versus laparotomy for ectopic pregnancy". Lancet; (1995) 345: 1139-1143.

13- Koruda, J.; Herbest, A.; and Reich, H.: "Laparoscopic Appendectomy". In: Reich \& Hulka (eds.) Textbook of Laparoscopy. 3rd ed.; (1998) 41: 491-496.

14- Semm, K. (ed.): Pelviscopy Operative Guidelines for Minimally Invasive Surgery. ISBN. (1992).

15- Cooper, C.S.; and Fisher, R.: "Predictors of laparoscopic complications after formal training in laparoscopic surgery". JAMA; (1993) 270: 2689-2692.

16- Peterson, H. B.; Hulka, J. F.; and Phillips, J. M.:
"American Association of Gynecologic Laparoscopists". 1988 Membership Survey on Operative Laparoscopy. J. Reprod. Med. (1990) 25 : 587.

17- Schwartz, R. O. : "Complications of laparoscopic hysterectomy". Obstet Gynaecol.; (1993) 81: 1022-1024.

18- Hulka, J. F.; Peterson, H. B.; and Phillips, M.: "Opcrative Laparoscopy-American Association of Gynecologic Laparoscopists 1991 Membership Survey". J. Reprod. Med; (1993) 38: 369-571.

19- Ohlgisser, M.; Sorokin, Y.; and Heifetz, M.: "Gynaecologic laparoscopy: a review article". Obstet Gynecol. Surv. (1985) 40: 385-396.

20- Garry $\mathrm{R}$,: towards evidence-based laparoscopic entry techniques: Clinical problems and dilemmas. Gynecol Endosc; (1999) 8: 315-326.

21- Kaali' S. G.; and Barad, D. H.: "Incidence of bowel injury due to dense adhesions at the site of direct trocar insertion". Reprod. Med.; (1992) 37: 617-618

22- Gomel, V.; and Taylor, P. (eds): "Complications and their management". In: Diagnostic and Operative Gynaecologic Laparoscopy. Mosby; (1992) 26: 306-307.

23- Redan, J. A. : "Emergency Department Laparoscopy". In: Hulka, F.; and Reich, H. (eds). textbook of Laparoscopy, 3rd Ed, Saunders Company; (1998) 42: 497-502.

24- Yuzpe, A. A. : "Pneumopertitoneum needle and trocar injuries in laparoscopy: a survery on possible contributing factors and prevention". J. reprod. Med.; (1990) 35: 485-490.

25- Eisemberg, J. M.: "Clinical economics. A guide to the economic analysis of clinical practice". JAMA; (1981) $262: 2879-86$. 